Matthias Bächle* and Fernando Puente León

\title{
Modeling of temperature dependency of structural waves in an ultrasonic flow measurement system
}

\author{
Modellierung der Temperaturabhängigkeit von Strukturschallwellen in einem \\ Ultraschall-Durchflussmesssystem
}

https://doi.org/10.1515/teme-2018-0067

Received October 1, 2018; accepted January 9, 2019

\begin{abstract}
Structural waves transmitted solely through the pipe wall influence the accuracy in a Clamp-On ultrasonic flow measurement system because of the superposition with the signals of interest. To improve the measurement against temperature variations, an algorithmic compensation of the structural waves using a temperature model is required. This paper proposes a temperature model for structural waves, using the Matching Pursuit method. In the first section, a sparse signal representation is presented to approximate the structural wave signals. The resulting signal coefficients are used to describe the temperature dependency in a linear model. The method is validated using measurements of structural waves in a circular pipe over a temperature range between $20^{\circ} \mathrm{C}$ and $80^{\circ} \mathrm{C}$. Based on these measurements, the accuracy of the approximated temperature model is evaluated and compared against the baseline signal-stretch method.
\end{abstract}

Keywords: Structural waves, ultrasound, modeling.

Zusammenfassung: Strukturschallwellen, welche sich nur über die Rohrwand ausbreiten, beeinflussen aufgrund der Überlagerung mit dem Nutzsignal die Genauigkeit in einem Clamp-On-Durchflussmesssystem. Um die Messung robust gegenüber Temperaturänderungen zu machen, wird eine algorithmische Kompensation der Strukturschallwellen mithilfe eines Temperaturmodells benötigt. In dieser Arbeit wird ein Temperaturmodell für Strukturschallwellen basierend auf der Matching-PursuitMethode vorgestellt. Im ersten Abschnitt wird eine geeignete Signaldarstellung eingeführt, um die Strukturschallwellen mit möglichst wenig Koeffizienten zu approximieren. Die Signalkoeffizienten werden verwendet, um die Temperaturabhängigkeit durch ein lineares Modell zu beschreiben. Die Methode wird anhand von Strukturschall-

*Corresponding author: Matthias Bächle, Karlsruhe Institute of Technology, Institute of Industrial Information Technology, Karlsruhe, Germany, e-mail: matthias.baechle@kit.edu

Fernando Puente León, Karlsruhe Institute of Technology, Institute of Industrial Information Technology, Karlsruhe, Germany messungen an einem Rohr über einem Temperaturbereich von $20^{\circ} \mathrm{C}$ bis $80^{\circ} \mathrm{C}$ validiert. Basierend auf diesen Messungen, wird die Genauigkeit des Temperaturmodells bestimmt und anschließend mit der Baseline-signal-stretchMethode verglichen.

Schlagwörter: Strukturschallwellen, Ultraschall, Modellierung.

\section{Introduction}

Ultrasonic flow meters (UFM) are increasingly popular due to the non-existence of moving parts, the response time and low energy consumption [6]. The working principles can be separated into the transit time method and the Doppler method. Using the transit time method is more popular because it does not require the presence of impurities in the fluids. Clamp-On systems, in which the ultrasonic pulses are excited through the pipe wall, are often preferable due to their non-intrusiveness. Furthermore, the sensors are protected from corrosive fluids and are easy to install [9]. However, the ultrasonic pulses propagate partly through the pipe walls as structural waves and partly through the fluids [1]. The part which solely propagates as structural waves can be regarded as correlated noise. This noise is a deterministic signal, which is interfering with the desired signal, and the accuracy of transit time measurements in ultrasonic flow measurement applications is highly dependent on the level of this noise $[10,12]$. For example, a superposition of sinusoidal desired signals with a sinusoidal noise signal at a signal-to-noise ratio of $20 \mathrm{~dB}$ leads, in the worst case, to a measurement error of $10 \%$.

An algorithmic approach to reduce the level of correlated noise is preferable, because it allows to save up constructive methods for suppressing the structural waves such as damping mats. One solution to this problem is to measure the structural waves before filling the pipes with water and then subtract the measured structural waves, but these waves are shown to be temperature dependent 
[2], which leads to wrong estimates of the structural waves during flow measurement.

The effects of temperature on structural waves in thin plates have also been extensively studied in the context of structural health monitoring [8, 5]. Dan et al. [3] extended the Rayleigh-Lamb equations (for further information refer to [11]) by variable coefficients to model the temperature effects and used the baseline signal-stretch (BSS) method to compensate them in a scenario, where the dominantly propagating wave was the So lamb wave. However, the problems of applicability in case of multiple reflection and multimode propagation still exist. Croxford et al. [2] quantitatively analyzed different compensation strategies using time shifts, multiple reference signals obtained at different temperatures and BSS. The models are applied after a separation into the different wave packets and can be formulated as

$$
u\left(t, T_{\mathrm{m}}\right) \approx \sum_{j} a_{j} s_{j}\left(t / \hat{\beta}-t_{j}\right) .
$$

The signal-stretch was performed using a resampling method and the influence of the quantization noise on the BSS has been examined. Harley and Moura [4] addressed the implementation of signal-stretching using the scale-transform domain to improve the computational efficiency.

Another aspect of structural waves analysis is the identification of different modes or temperature effects. For this purpose $\mathrm{Xu}$ et al. [13] used the Matching Pursuit (MP) algorithm with a Gabor dictionary and a chirplet dictionary and showed that the propagation mode can be identified by evaluating the sign of the chirp rate. Extending the MP algorithm by a second constrained MP algorithm, Lu and Michaels [7] could differentiate between signal changes due to temperature variation and damage. Although the combination of two MP algorithms allows an identification of temperature variation, an exact estimation of signals at different temperatures has not been researched.

In this paper we propose a new method to model the temperature dependency of structural waves in a multimode and multipath scenario. The method is based on a signal decomposition using the MP algorithm and a subsequent constraint MP algorithm. The signal decomposition results in a set of parameters. We show that in the new parameter space we can approximate signals at temperatures which are in-between the initial measurement temperatures by using a linear interpolation. Using the method reduces the necessary structural waves measurements and the transformation parameters can be directly calculated from the temperature. This paper is organized as follows. In the second section, the used MP algorithm is presented. The extension of the existing MP approach to compensate varying temperatures is shown in Section 3. Finally, the method is validated and compared against signal-stretch based methods using measured signals from a Clamp-On system in a circular pipe.

\section{Signal representation}

The first step is to decompose the measurement signal using the MP algorithm. In this section, a short introduction into the algorithm and an extension to use constraints is given. The aim is to transform the signal $s(t)$ in a sparse representation, meaning that in the transformed signal representation the signal energy is compressed in as few as possible coefficients $a_{k}$. Coefficients which are nearly zero can be omitted. MP tries to approximate a signal as weighted sum of functions:

$$
s(t) \approx \hat{s}(t)=\sum_{k} a_{k} g_{k}(t) .
$$

Therefore, the best functions $g_{k}(t)$ are chosen from an overcomplete function set $\mathcal{D}=$ Frame $\left\{g_{k}(t), k=-\infty, \ldots, \infty\right\}$ such that given a constant $K$ the optimization problem

$$
\text { minimize }\left\|s(t)-\sum_{k=1}^{K} a_{k} g_{k}(t)\right\|_{2}^{2} \quad \text { w.r.t. } \quad g_{k}(t) \in \mathcal{D}
$$

is solved. The MP algorithm does not provide an optimal solution to (3). It is based on a greedy algorithm which always chooses the locally optimal function in which optimality is defined as the function with the biggest inner product with the residual signal $r_{i}(t)$ at the iteration $i$. The iterative MP can be formulated as follows [13]:

$$
\left\{\begin{array}{l}
r_{0}(t)=s(t), \\
g_{i}(t)=\arg \max _{g_{k}(t) \in \mathcal{D}}\left|\left\langle r_{i}(t), g_{k}(t)\right\rangle\right|, \\
a_{i}=\left\langle r_{i}(t), g_{i}(t)\right\rangle, \\
r_{i+1}(t)=r_{i}(t)-a_{i} \cdot g_{i}(t) .
\end{array}\right.
$$

After every iteration the residual signal energy is reduced. The iterations are continued until a termination criterion is reached. This can be either a maximum number of iterations or a minimum residual signal energy.

Due to the narrowband characteristic of ultrasonic signals, Gabor wavelets with variable time delays $t_{k}$, modulation frequencies $f_{k}$ and time duration $\sigma_{k}$ are chosen to 
build the frame:

$$
g_{k}(t)=c_{k} \exp \left(-\frac{\left(t-t_{k}\right)^{2}}{2 \sigma_{k}^{2}}+\mathrm{j} 2 \pi f_{k} t\right)
$$

with $c_{k}$ subject to

$$
\left\langle g_{k}(t), g_{k}(t)\right\rangle=1
$$

Equation (4) implies that the usage of complex Gabor wavelets results in complex projections, which is not wanted, because the input signals are real and the residuals should be real as well. As in the Fourier transform, this could be solved by using the positive and negative frequency components of the same wavelet. However, it is simpler to take the real part of the projection. Thus, the update rule (4) is changed to

$$
r_{i+1}(t)=r_{i}(t)-\operatorname{Re}\left\{\left\langle r_{i}(t), g_{i}(t)\right\rangle \cdot g_{i}(t)\right\} .
$$

Simple usage of the trigonometric relations can show that the update rule (7) in connection with the Gabor wavelet (5) as $g_{i}(t)$ is equivalent to using the update rule

$$
r_{i+1}(t)=r_{i}(t)-\left\langle r_{i}(t), g_{i}(t)\right\rangle \cdot g_{i}(t)
$$

with the frame function

$$
g_{i}(t)=c_{i} \exp \left(-\frac{\left(t-t_{i}\right)^{2}}{2 \sigma_{i}^{2}}\right) \cdot \cos \left(2 \pi f_{i} t+\phi\right)
$$

and the optimal phase parameter $\phi$, as done by Xu et al. [13]. Therefore, by using the complex Gabor wavelet (5), the phase parameter $\phi$ at iteration $i$ can be calculated directly by

$$
\phi=\arctan \left(\frac{\operatorname{Im}\left\{\left\langle r_{i}(t), g_{i}(t)\right\rangle\right\}}{\operatorname{Re}\left\{\left\langle r_{i}(t), g_{i}(t)\right\rangle\right\}}\right)
$$

and is automatically optimal. Lastly, due to the normalization (6), we have to add the factor two in the update rule to compensate that the energy is halved by only taking the real part:

$$
r_{i+1}(t)=r_{i}(t)-2 \cdot \operatorname{Re}\left\{\left\langle r_{i}(t), g_{i}(t)\right\rangle \cdot g_{i}(t)\right\} .
$$

The problem that requires the most computational effort is finding the best function in $\mathcal{D}$. Suppose the functions are built from a model $g_{k}(t)=g(\boldsymbol{\theta}, t)$ with a parameter vector $\boldsymbol{\theta} \in \mathbb{R}^{P}$. If every parameter is discretized in $N$ steps, then a brute force search would need to calculate $N^{P}$ inner products. Therefore, in this work we use an optimization method to solve a constrained optimization problem. This reduces the computing time and at the same time allows a high resolution of the parameter space.
Additionally, we extend the optimization problem to use constraints. This way, we can force the decomposition to approximate the signals only in the time ranges of interest. Furthermore, we can limit the duration $\sigma_{k}$ and modulation frequencies $f_{k}$ of the best matching functions $g_{k}(t)$ to have physically meaningful values. If constraints for the parameters in (5) are used, the optimization problem in (4) changes to

$$
\left[t_{k}, f_{k}, \sigma_{k}\right]=\arg \max _{t_{k}, f_{k}, \sigma_{k}} Q\left(\left[t_{k}, f_{k}, \sigma_{k}\right]\right)
$$

with the quality function

$$
\begin{aligned}
Q\left(\left[t_{k}, f_{k}, \sigma_{k}\right]\right)= & \left|\left\langle r_{i}(t), g\left(t,\left[t_{k}, f_{k}, \sigma_{k}\right]\right)\right\rangle\right| \\
& +c_{\mathrm{f}} \cdot\left(f_{k}-f_{\mathrm{set}}\right)^{2}+c_{\sigma} \cdot\left(\sigma_{k}-\sigma_{\mathrm{set}}\right)^{2} \\
& +c_{\mathrm{t}} \cdot\left(t_{k}-t_{\mathrm{set}}\right)^{2} \\
& +c_{\mathrm{t}, \max } \cdot\left(t_{k}-t_{\mathrm{max}}\right)^{2} \cdot h\left(t_{k}-t_{\mathrm{max}}\right),
\end{aligned}
$$

and $h(t)$ denoting the Heaviside step function. The constraints can be regulated by the multipliers $c_{\mathrm{f}}, c_{\sigma}, c_{\mathrm{t}}$, which allows using different combinations of constraints. Using $c_{\mathrm{t}, \max }$, the MP algorithm can be restricted to a maximal time range, but restricting the maximal time delays may lead to suboptimal solutions due to the occurrence of local minima, which can lead to convergence problems of the MP. Therefore, the parameter $c_{\mathrm{t}, \max }$ was tuned to be very small and all the solutions that violated the constraint were used to minimize the residuum, but were not saved to be used for the temperature model.

\section{Temperature model}

In the following, the temperature model using the MP decomposition is presented. Given two measurements $s\left(t, T_{0}\right)$ and $s\left(t, T_{\mathrm{m}}\right)$ recorded at two different temperatures $T_{0}$ and $T_{\mathrm{m}}$, the model tries to estimate the signals for temperatures $T \in\left[T_{0}, T_{\mathrm{m}}\right]$. The idea of the model is pictured in Fig. 1. It consists of two MP decompositions: First the baseline signal $s\left(t, T_{0}\right)$ is decomposed into $K$ coefficients $a_{k}$ and $K$ functions $g_{k}(t)$. In the following, this is called

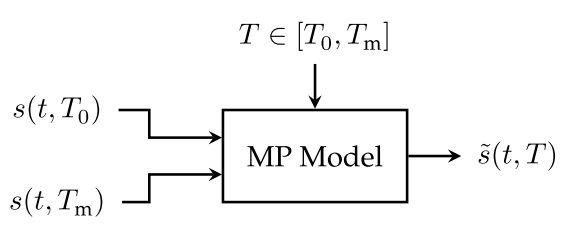

Figure 1: Matching Pursuit model. 
the baseline Matching Pursuit (BMP). As stopping criterion, the residual signal energy in the desired time range has to be damped below a configurable value. The second step is to decompose the signal $s\left(t, T_{\mathrm{m}}\right)$ using a constraint MP, in which the time delays $t_{k}$ resulting from the decomposition of $s\left(t, T_{0}\right)$ are used as start values for the optimization algorithm and the number of coefficients is kept constant. This is done to guarantee the same decomposition order, which is necessary because the functions $g_{k}(t)$ are not orthogonal. Furthermore, to improve robustness, some of the model parameters $\sigma_{k}$, $f_{k}$ or $t_{k}$ can be kept constant using the constraints given in (12) to reduce the degrees of freedom. In the following, the second MP decomposition is called constraint Matching Pursuit (CMP).

The frame used in the BMP can have three degrees of freedom. The combinations of the different free parameters are summarized in following set:

$$
\begin{aligned}
\mathcal{M}_{\mathrm{BMP}} & =\left\{\left[t_{k}, f_{k}, \sigma_{k}\right],\left[t_{k}, f_{k}\right], t_{k}\right\} \\
& =\left\{\mathbf{m}_{1, \mathrm{BMP}}, \mathbf{m}_{2, \mathrm{BMP}}, \mathbf{m}_{3, \mathrm{BMP}}\right\} .
\end{aligned}
$$

In a frame with less degrees of freedom, the fixed time duration of the frame functions was set to $\sigma_{k}=t_{\max } / 20$. This way, an acceptable adaptability to different wave packets can be attained. By setting the fixed modulation frequencies $f_{k}$ to the excitation frequency $f_{0}$, the frame functions are adapted to the expected structural waves. Mathematically, this is equivalent to setting $c_{\mathrm{f}} \rightarrow \infty$ or $c_{\sigma} \rightarrow \infty$ in (12). Nevertheless, even if the set $\mathbf{m}_{1, \text { BMP }}$ is used, excessive deviations from the excitation frequency $f_{0}$ or time duration $t_{\max } / 20$ are prevented using moderate values for $c_{\mathrm{f}}$ and $c_{\sigma}$. The maximum for the time delays $t_{k}$ is also kept.

After the BMP of $s\left(t, T_{0}\right)$ we have a set of parameters $\theta=\left\{\left[a_{k}, t_{k}, f_{k}, \sigma_{k}\right], k \in[1, K]\right\}$. Subsequently, the parameters $t_{k}, f_{k}, \sigma_{k}$ are used to reduce the search space in the optimization problem (12) during each iteration. Following the same order as the decomposition in the BMP, the time durations $\sigma_{k}$ are fixed and $t_{k}, f_{k}$ are either used as starting values of the optimization algorithm or kept constant. This results in different grades of freedom, which are described by the set

$$
\begin{aligned}
\mathcal{M}_{\mathrm{CMP}} & =\left\{\left[t_{k}, f_{k}\right], f_{k}, t_{k}, \emptyset\right\} \\
& =\left\{\mathbf{m}_{1, \mathrm{CMP}}, \mathbf{m}_{2, \mathrm{CMP}}, \mathbf{m}_{3, \mathrm{CMP}}, \mathbf{m}_{4, \mathrm{CMP}}\right\} .
\end{aligned}
$$

The empty set $\mathbf{m}_{4, \mathrm{CMP}}$ represents a fully constricted decomposition, meaning that no optimization is necessary.

After the BMP of $s\left(t, T_{0}\right)$ and the CMP of $s\left(t, T_{\mathrm{m}}\right)$, we have two sets of parameters $\theta_{\mathrm{BMP}}$ and $\theta_{\mathrm{CMP}}$. The linear in-

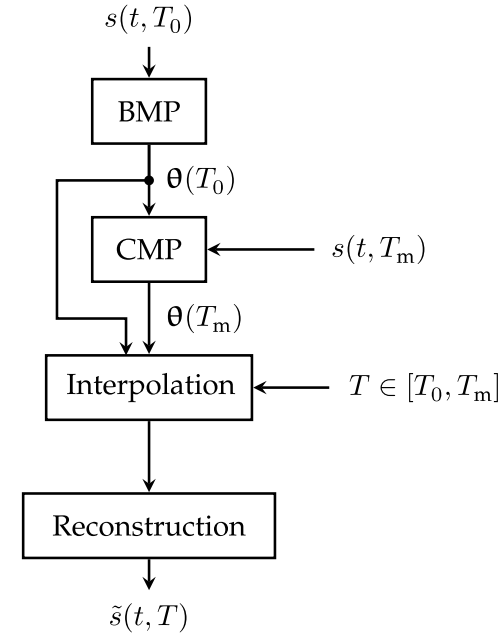

Figure 2: Flowchart of the MP method.

terpolation in the parameter space can be formulated as

$$
\begin{aligned}
\tilde{t}_{k}(T)= & \left(t_{k, \mathrm{CMP}}-t_{k, \mathrm{BMP}}\right) \cdot p(T)+t_{k, \mathrm{BMP}} \\
\tilde{f}_{k}(T)= & \left(f_{k, \mathrm{CMP}}-f_{k, \mathrm{BMP}}\right) \cdot p(T)+f_{k, \mathrm{BMP}} \\
\left|\tilde{a}_{k}(T)\right|= & \left(\left|a_{k, \mathrm{CMP}}\right|-\left|a_{k, \mathrm{BMP}}\right|\right) \cdot p(T)+\left|a_{k, \mathrm{BMP}}\right| \\
\arg \left(\tilde{a}_{k}\right)(T)= & \left(\arg \left(a_{k, \mathrm{CMP}}\right)-\arg \left(a_{k, \mathrm{BMP}}\right)\right) \cdot p(T) \\
& +\arg \left(a_{k, \mathrm{BMP}}\right)
\end{aligned}
$$

with

$$
p(T)=\frac{T-T_{0}}{T_{\mathrm{m}}-T_{0}} \quad, \quad k \in\{1, \ldots, K\} .
$$

Parameters which have been kept constant are not interpolated and the complex coefficients are reconstructed from the interpolated absolute values and phase information. Finally, a reconstruction of the signals from the parameter space is necessary. This is done by the weighted sum

$$
\tilde{s}(t, T)=\sum_{k=1}^{K} \tilde{a}_{k}(T) g\left(\tilde{t}_{k}, \tilde{f}_{k}, t ; T\right) .
$$

The overview of the algorithm is depicted in Fig. 2. Summarized, the algorithm can be described as follows. The first step is the BMP of the baseline signal $s\left(t, T_{0}\right)$. Using the parameters from the BMP, the CMP is performed on the signal $s\left(t, T_{\mathrm{m}}\right)$. The estimated signal is then reconstructed from the interpolated parameters using (16) and (18).

\section{Experimental results}

To validate the model, in a multimode and multipath propagation scenario structural wave signals were recorded at 


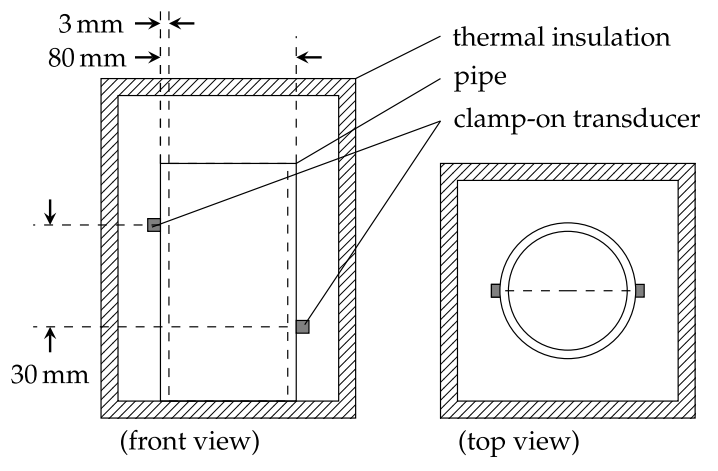

Figure 3: Experimental setup.

different temperatures. An experimental stand consisting of a circular pipe with piezoelectric Clamp-On transducers attached was erected and thermally insulated. The pipe was $0.5 \mathrm{~m}$ long, made of stainless steel and filled with air, to validate only the structural waves. The measurement signals were acquired at a sample rate of $f_{\mathrm{s}}=50 \mathrm{MHz}$ using a preamplifier and a PXIe-1062 station with a PXIe-5171 ADC module and a PXI-5412 DAC module. The measurement time was set to $150 \mu \mathrm{s}$, to avoid reflections from the ends of the pipe. Nevertheless, the waves have different propagation paths due to multiple circulations of the wave packets resulting from the circular form of the pipe. A scheme of the experiment is depicted in Fig. 3. As excitation signal a gaussian modulated cosine signal

$$
u(t)=A \cdot \exp \left(-\frac{\left(t-t_{\mathrm{u}}\right)^{2}}{2 \sigma^{2}}\right) \cdot \cos \left(2 \pi f_{0} t\right)
$$

has been used, with $t_{\mathrm{u}}=5 \mu \mathrm{s}, \sigma=1.5 \mu \mathrm{s}$ and $A=10 \mathrm{~V}$. Due to the excitation frequency, which was set to $700 \mathrm{kHz}$, and the material thickness, primarily $S_{0}$ and $A_{0}$ modes are propagating. The pipe was first heated to a temperature of about $80^{\circ} \mathrm{C}$ and then thermally insulated using styrofoam. The natural cooling-off to $20^{\circ} \mathrm{C}$ took 4 hours. During this time window 72200 measurement signals and their corresponding temperatures were recorded. To reduce electronic noise, the signals were packaged in blocks of 100 and averaged, which resulted in 722 signals.

The first measurement signal from the temperature experiment and its residuals after increasing number of MP iterations is shown in Fig. 4. The residual signal energy is reduced to less than $-25 \mathrm{~dB}$ after only 14 iterations, but to reach $-40 \mathrm{~dB} 60$ iterations are needed. Further increasing the damping setpoints does not further improve the temperature model, but the computational effort increases strongly. The BMP was configured to reduce the residual by $40 \mathrm{~dB}$ in the time range $[0 \mu \mathrm{s}, 100 \mu \mathrm{s}]$. Therefore, the residuals after $100 \mu$ s are the same as the original signal. It is

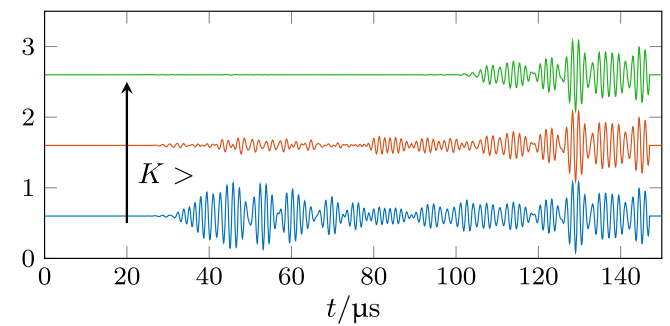

Figure 4: Original measurement signal (blue, bottom) and the residuals from MP after 7 (red, middle) and 60 iterations (green, top).

shown that by limiting the time delays and the width $\sigma_{k}$ of the frame functions, the MP model can be used to describe local signal distortions. For example, in Fig. 4 only the temperature dependency of signal parts arriving in $[0 \mu \mathrm{s}, 100 \mu \mathrm{s}]$ are considered. This shows that the method can deal with signals where a temperature variation has different effects on the different propagation paths.

Using the residual signal energy normalized by the original signal energy

$$
J(T)=\frac{\|\tilde{s}(t, T)-s(t, T)\|_{2}^{2}}{\|s(t, T)\|_{2}^{2}},
$$

the model performance was evaluated by comparison of the interpolated signals with the measured signal in the desired time range $t \in\left[t_{0}, t_{N-1}\right]$. Smaller values of $J(T)$ represent better model performance. We examined the influence of the degrees of freedom of MP model, the different time ranges as well as different temperature differences $\Delta T=T_{\mathrm{m}}-T_{0}$. The lower temperature $T_{0}$ is always set to $20^{\circ} \mathrm{C}$ and the lower limit of the time range is alway set to $t_{0}=0 \mathrm{~s}$.

To show that an interpolation in the parameter space is valid, the optimal parameters were calculated for every temperature $T \in\left[20^{\circ} \mathrm{C}, 81^{\circ} \mathrm{C}\right]$. The function set for decomposition has been set by BMP of the baseline signal $s\left(t, T_{0}\right)$ with the free parameters $t_{k}, f_{k}, \sigma_{k}$. Subsequently CMP with zero degrees of freedom was used on the signals at higher temperatures to determine the optimal coefficients $a_{k}(T)$. Figure 5 presents the results of this analysis. In the upper plot the attained model performance is shown, which is the optimum under the configured constraints. The associated optimal parameters $a_{k}$ are shown in the middle plot (absolute value) and in the bottom plot (phase). For the purpose of better presentation and clarity only the parameters of the four frame functions with the highest signal energy are plotted. The phase of the coefficients $a_{k}$ show a linear behavior, while their absolute values only show a small deviation from linearity. The jumps of $\arg \left(a_{k}\right)$ at $T=55^{\circ} \mathrm{C}$ and $T=72^{\circ} \mathrm{C}$ arise through the $2 \pi$ indeterminacy 

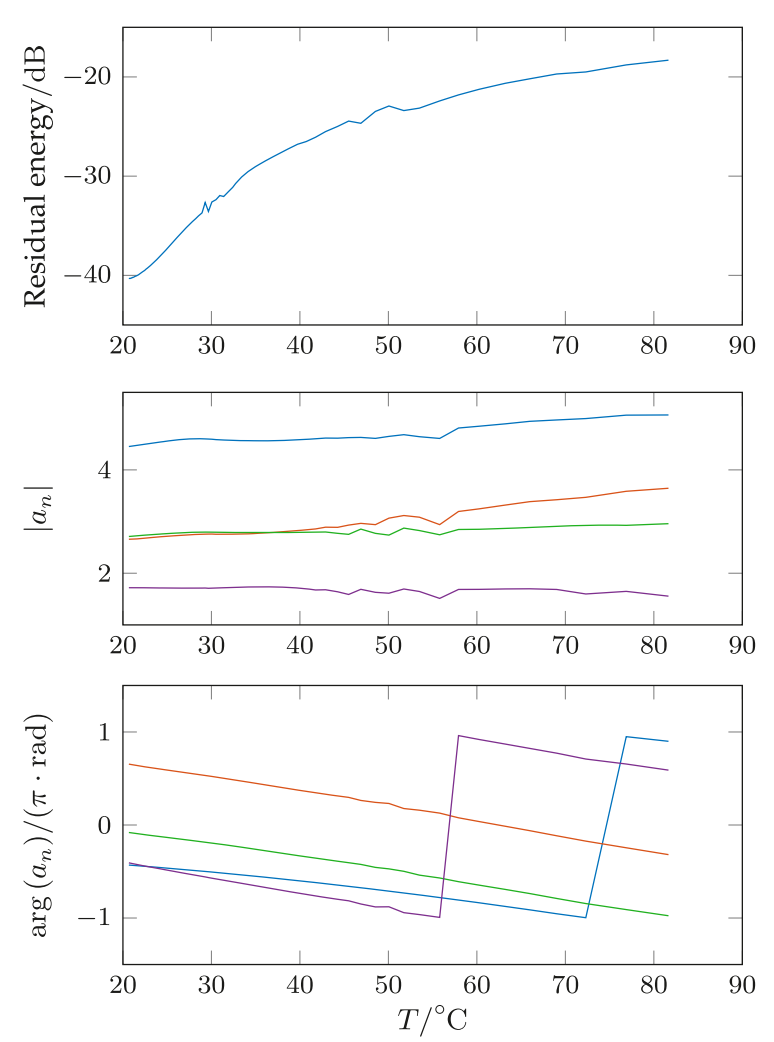

Figure 5: Upper limit of model performance (top) and parameter trend of CMP over increasing temperature: absolute values (middle) and phase information (bottom). The four curves are the four coefficients with the largest absolute value.

of the phase. One jump per coefficient can be solved by the assumption that the trend of $\arg \left(a_{k}\right)$ has to be monotonically decreasing with increasing temperature. To solve more than one jump, another measurement with smaller temperature difference would be needed. In the range of $\left[45^{\circ} \mathrm{C}, 60^{\circ} \mathrm{C}\right]$ inconsistencies can be observed. They also show up in the model performance of the MP method and the BSS method. Possible reasons can be disturbances during the experiment or the influence of the temperature on the transducers or their coupling.

In the following, the model is compared with the BSS method presented in [2]. In this work the model is extended by an amplification factor to deal with temperature-dependent damping of the transducers. The estimated signals are calculated by

$$
\tilde{s}_{\mathrm{BSS}}(t, T)=\tilde{A}(T) \cdot s\left(\tilde{\alpha}(T) \cdot t, T_{0}\right) .
$$

The temperature-dependent parameters $\tilde{A}(T)$ and $\tilde{\alpha}(T)$ are determined by linear interpolation

$$
\begin{gathered}
\tilde{A}(T)=\left(\hat{A}\left(T_{\mathrm{m}}\right)-1\right) \cdot p(T)+1, \\
\tilde{\alpha}(T)=\left(\hat{\alpha}\left(T_{\mathrm{m}}\right)-1\right) \cdot p(T)+1,
\end{gathered}
$$

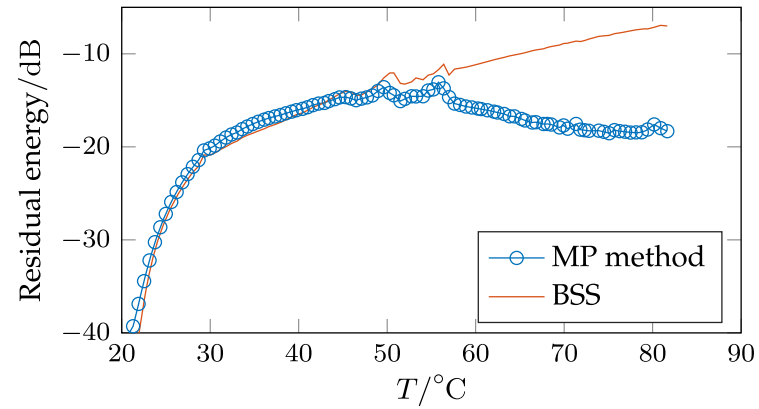

Figure 6: Residual signal energy of temperature model with $t_{N-1}=100 \mu \mathrm{s}$. The degrees of freedom on the BMP have been set to $\mathrm{m}_{1, \mathrm{BMP}}=\left[\sigma_{k}, f_{k}, t_{k}\right]$ The CMP had zero degrees of freedom.

with

$$
\left[\begin{array}{l}
\hat{A}\left(T_{\mathrm{m}}\right) \\
\hat{\alpha}\left(T_{\mathrm{m}}\right)
\end{array}\right]=\arg \min _{\{A, \alpha\}} \sum_{n=0}^{N-1}\left(s\left(t_{n}, T_{\mathrm{m}}\right)-A \cdot s\left(\alpha t_{n}, T_{0}\right)\right)^{2} .
$$

The time-stretch is done by resampling as described in [2] with a high amount of zero padding such that granularization noise is lower than expected model errors. Using equation (21) and the MP method, the signals $\tilde{s}(t, T)$ are now estimated from the baseline signal $s\left(t, T_{0}\right)$ and the signal $s\left(t, T_{\mathrm{m}}\right)$. Figure 6 shows the residual energy level using (20), with the temperature $T_{\mathrm{m}}$ set to the maximum and the time range set to $[0 \mathrm{~s}, 100 \mu \mathrm{s}]$. The BSS method's performance decreases with increasing temperature, because applying a constant amplification and time-stretch cannot deal with signal distortions such as the shapes of the envelopes. Croxford et al. called this frequency noise [2]. Note that the BSS method has less free parameters, so the adaption to signal changes is naturally limited. Because the stopping criterion of the MP was set to $40 \mathrm{~dB}$, the accuracy of the MP method is limited to this damping, even if there are no temperature variations at all (see Fig. 6). The performance of the MP method is also decreasing, because the constraints in the CMP limit the adaptability. The point with least damping $\left(T \approx 50^{\circ} \mathrm{C}\right)$ is due to the combination of the interpolation errors of the parameters and the limited adaptability due to the constraints. These results show that for small temperature deviations from the boundary $T_{0}$ the BSS method works better and for temperature deviations $T-T_{0}>30 \mathrm{~K}$, the MP method is preferable. This temperature should not be confused with the difference of the boundary temperatures $T_{\mathrm{m}}-T_{0}$ between the signal estimation is to be done (see Fig. 1).

In the following, the influence of the constraints and the chosen dictionary is examined. Therefore the MP method has been run several times with the different constraints $\mathcal{M}_{\mathrm{BMP}}$ and $\mathcal{M}_{\mathrm{CMP}}$ under the conditions $\Delta T=60 \mathrm{~K}$ 
Table 1: Influence of constraints on model performance at $t_{N-1}=$ $100 \mu \mathrm{s}$ and $\Delta T=60 \mathrm{~K}$.

\begin{tabular}{lrrr}
\hline $\mathcal{M}_{\mathrm{CMP}} \backslash \mathcal{M}_{\mathrm{BMP}}$ & $\boldsymbol{t}_{\boldsymbol{k}}, \boldsymbol{f}_{\boldsymbol{k}}, \boldsymbol{\sigma}_{\boldsymbol{k}}$ & $\boldsymbol{t}_{\boldsymbol{k}}, \boldsymbol{f}_{\boldsymbol{k}}$ & $\boldsymbol{t}_{\boldsymbol{k}}$ \\
\hline$m_{1, \mathrm{CMP}}=\left[t_{k}, f_{k}\right]$ & $52.63 \mathrm{~dB}$ & $24.63 \mathrm{~dB}$ & $21.58 \mathrm{~dB}$ \\
$m_{2, \mathrm{CMP}}=f_{k}$ & $-13.43 \mathrm{~dB}$ & $-10.73 \mathrm{~dB}$ & $-14.21 \mathrm{~dB}$ \\
$m_{3, \mathrm{CMP}}=t_{k}$ & $5.00 \mathrm{~dB}$ & $4.80 \mathrm{~dB}$ & $4.59 \mathrm{~dB}$ \\
$m_{4, \mathrm{CMP}}=\emptyset$ & $-13.05 \mathrm{~dB}$ & $-14.28 \mathrm{~dB}$ & $-13.67 \mathrm{~dB}$ \\
\hline
\end{tabular}

and $t_{N-1}=100 \mu \mathrm{s}$. The points of least performance are calculated for the different constraints by

$$
J\left(\mathcal{M}_{\mathrm{BMP}}, \mathcal{M}_{\mathrm{CMP}}\right)=\max _{T} J\left(\mathcal{M}_{\mathrm{BMP}}, \mathcal{M}_{\mathrm{CMP}}, T\right)
$$

and displayed in Table 1. Positive values represent a bad performance. The results show that the choice of constraints in the CMP is very important, as using $\mathbf{m}_{1, \mathrm{CMP}}$ and $\mathbf{m}_{3, \text { CMP }}$ does not lead to a satisfactory signal estimation. The reason lies in the optimization procedures which calculate the parameters. If the parameter $t_{k}$ can be adapted, the optimization may find a completely different frame function in one step, and therefore lead to a different MP decomposition. To apply the MP method, the signal decompositions of $s\left(t, T_{0}\right)$ and $s\left(t, T_{\mathrm{m}}\right)$ have to be related. Table 1 shows this correlation to be only possible, if the parameter $t_{k}$ is constrained to the same value as in the BMP of $s\left(t, T_{0}\right)$. Due to these results, in the following evaluations of the method, the constraints of the BMP and the CMP have been set to $\mathbf{m}_{1, \mathrm{BMP}}$ and $\mathbf{m}_{4, \mathrm{CMP}}$, respectively.

After evaluation of the MP method with a constant time range of $[0 \mu \mathrm{s}, 100 \mu \mathrm{s}]$ and maximal temperature difference $\Delta T$, in Fig. 7 the influence of the temperature difference $\Delta T$ is shown. This is done by repeating the MP method with decreasing $\Delta T$. The model performance was consequently only evaluated for temperatures $T \in\left[T_{0}, T_{0}+\Delta T\right]$. In Figure 7 the worst case for the respective $\Delta T$ is depicted following the equation:

$$
J\left(\Delta T=T_{\mathrm{m}}-T_{0}\right)=\max _{T \in\left[T_{0}, T_{\mathrm{m}}\right]} J\left(T ; T_{\mathrm{m}}\right) .
$$

The worst damping of the MP method is better than the BSS method for nearly every $\Delta T$. However, the BSS method can attain better signal estimations at small temperature variations, because the MP method cannot estimate the signal better than the predefined stopping criterion of the MP algorithm.

Figure 8 shows the dependency of the MP method on the time range in which the signals should be estimated. Wave packets arriving later correspond to modes which propagate with a lower group velocity or to wave packets with a longer propagation path. The performance of

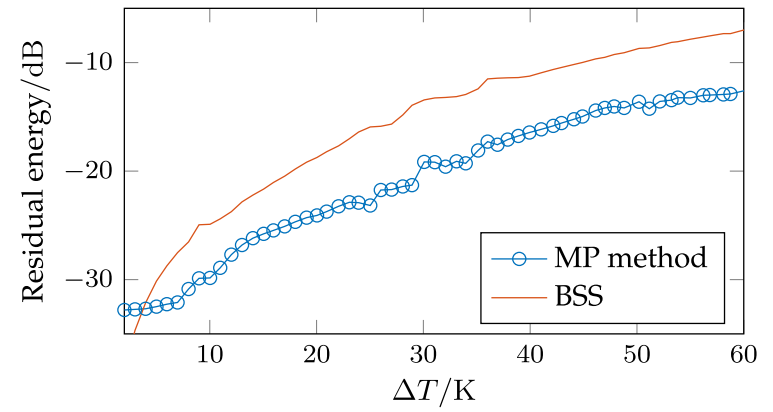

Figure 7: Attained model performance under varied temperature difference $\Delta T$ (BMP set to $\mathrm{m}_{1, \mathrm{BMP}}$, CMP set to $\mathrm{m}_{4, \mathrm{CMP}}$ ).

the BSS method decreases for later wave packets, because the stretch factor is only globally determined and cannot be adapted individually for every wave packet. However, Fig. 8 shows that the performance of the MP method also decreases if the time range is increased. The reason for this behavior is the linear interpolation to estimate the parameters. Later wave packets are more dispersive and overlap with wave packets that propagate on a shorter path but arise from the reverberating transducer. This results in an increasingly nonlinear relationship of the parameters with the temperature and therefore reduces model performance.

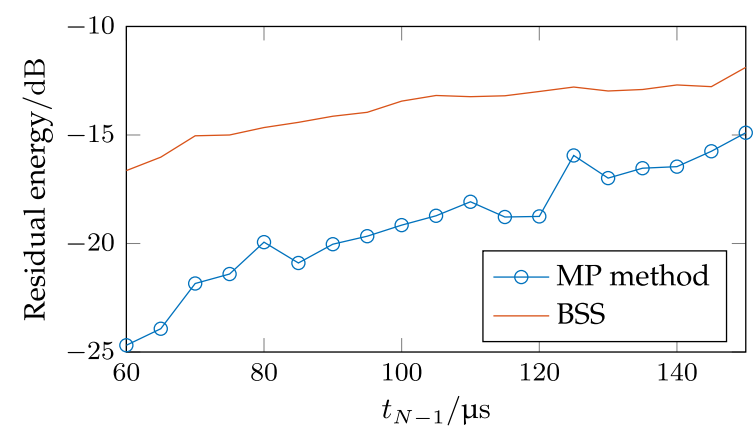

Figure 8: Attained model performance under varied time range $\Delta T$ (BMP set to $\mathbf{m}_{1, \mathrm{BMP}}$, CMP set to $\mathbf{m}_{4, \mathrm{CMP}}$ ).

\section{Conclusions}

This paper investigated the application of the Matching Pursuit (MP) algorithm for modeling the temperature dependency of structural waves. To this end, two specially designed MP algorithms were developed, called the baseline Matching Pursuit (BMP) and the constrained Matching Pursuit (CMP). The numerical implementation was 
described as well as the extensions to use constraints. The results of different degrees of freedom in the BMP and CMP were investigated and compared against the baseline signal-stretch (BSS) method. In the CMP the time delays $t_{k}$ needed to be kept constant, because otherwise the correlation between the parameters of BMP and CMP was not possible. The parameters were shown to have a fairly linear relation to the temperature. A linear interpolation of the parameters to estimate the temperature influence on the signals reached similar residual signal energies as the BSS method in the temperature range $\left[20^{\circ} \mathrm{C}, 50^{\circ} \mathrm{C}\right]$. At temperature variations $\Delta T>30^{\circ} \mathrm{C}$, the MP method performed better and yielded a signal damping of at least $-13 \mathrm{~dB}$.

Furthermore, the influence of different temperature differences and time ranges on the performance of the method have been investigated. For temperaturedifferences greater than $10^{\circ} \mathrm{C}$, the MP method could estimate the temperature dependent signal better than the BSS method by about $5 \mathrm{~dB}$. Increasing time ranges impair the estimation accuracy for both the MP method and the BSS method, but at the maximal time range examined, the MP method still could approximate the signals better.

Using this approach in an ultrasonic flow measurement system to compensate the structural waves, an improvement of the robustness against temperature variations is to be expected. Without a compensation of the structural waves, the accuracy of flow estimation is dependent on the phase shift of the structural waves compared to the desired signal. Therefore, performing an experiment with constant flow, over varying temperature, a ripple in the accuracy over the temperature can be observed, due to the varying phase shift. Using our compensation approach, the peak-to-peak value of this ripple is expected to get smaller.

In order to validate the compensation of the structural waves using the MP model, the setup of an experimental stand, which allows high temperature variations during flow measurements, is necessary. Furthermore, the application of wavelet packets instead of the Gabor wavelet based Matching Pursuit method and the problem of nonlinearity in later wave packets are subjects of future research.

\section{References}

1. M. Aanes, R. A. Kippersund, K. D. Lohne, K.-E. Frøysa, and P. Lunde. Time-of-flight dependency on transducer separation distance in a reflective-path guided-wave ultrasonic flow meter at zero flow conditions. The Journal of the Acoustical Society of America, 142 (2): 825-837, 2017.
2. A. J. Croxford, J. Moll, P. D. Wilcox, and J. E. Michaels. Efficient temperature compensation strategies for guided wave structural health monitoring. Ultrasonics, 50 (4): 517-528, 2010.

3. C. A. Dan, P. Kudela, and W. Ostachowicz. Compensation of temperature effects on guided wave based structural health monitoring systems. In EWSHM-7th European Workshop on Structural Health Monitoring, 2014.

4. J.B. Harley and J. M. Moura. Scale transform signal processing for optimal ultrasonic temperature compensation. IEEE transactions on ultrasonics, ferroelectrics, and frequency control, 59 (10): 2226-2236, 2012.

5. F. Lanza di Scalea and S. Salamone. Temperature effects in ultrasonic lamb wave structural health monitoring systems. The Journal of the Acoustical Society of America, 124 (1): 161-174, 2008.

6. Y. Lin. Signal processing and experimental technology in ultrasonic flow measurement. Phd dissertation, University of Duisburg, 2004.

7. Y. Lu and J. E. Michaels. Numerical implementation of matching pursuit for the analysis of complex ultrasonic signals. IEEE transactions on ultrasonics, ferroelectrics, and frequency control, 55 (1): 173-182, 2008.

8. A. Raghavan and C. E. Cesnik. Effects of elevated temperature on guided-wave structural health monitoring. Journal of Intelligent Material Systems and Structures, 19 (12): 1383-1398, 2008.

9. G. Rajita and N. Mandal. Review on transit time ultrasonic flowmeter. In 2016 2nd International Conference on Control, Instrumentation, Energy \& Communication (CIEC), pages 88-92, 2016.

10. N. Roosnek. Novel digital signal processing techniques for ultrasonic gas flow measurements. Flow Measurement and Instrumentation, 11 (2): 89-99, 2000.

11. J. L. Rose. Ultrasonic Waves in Solid Media. Cambridge University Press, Cambridge, 1999.

12. S. J. Rupitsch. Piezoelectric Sensors and Actuators. Springer-Verlag Berlin Heidelberg, Heidelberg, 2018.

13. B. Xu, V. Giurgiutiu, and L. Yu. Lamb waves decomposition and mode identification using matching pursuit method. In Sensors and Smart Structures Technologies for Civil, Mechanical, and Aerospace Systems 2009. International Society for Optics and Photonics, 2009.

\section{Bionotes}

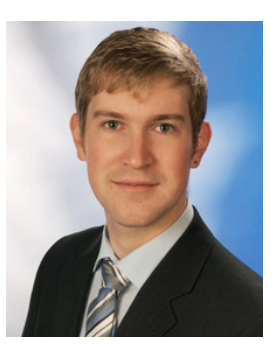

\section{Matthias Bächle}

Karlsruhe Institute of Technology, Institute of Industrial Information Technology, Karlsruhe, Germany matthias.baechle@kit.edu

Matthias Bächle obtained his bachelor's and master's degree in Electrical Engineering/Information Technology at the Karlsruhe 
Institute of Technology, Germany in 2014 and 2016, respectively. $\mathrm{He}$ is currently working as a research associate at the Institute of Industrial Information Technology (IIIT) at the Karlsruhe Institute of Technology, Germany, where he is pursuing his Ph.D. degree. His current research interests include signal processing techniques and their application to flow measurement using transit time of ultrasonic pulses.

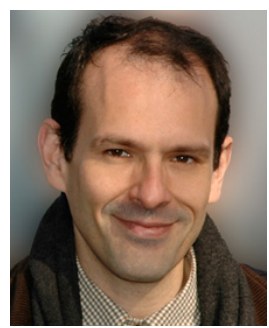

\section{Fernando Puente León}

Karlsruhe Institute of Technology, Institute of Industrial Information Technology, Karlsruhe, Germany

fernando.puente-leon@kit.edu

Fernando Puente León is a Professor with the Department of Electrical Engineering and Information Technology at Karlsruhe Institute of Technology, Germany, where he heads the Institute of Industrial Information Technology (IIIT). From 2001 to 2002, he was with DS2, Valencia, Spain. From 2002 to 2003, he was a Postdoctoral Research Associate with the Institut für Mess- und Regelungstechnik, University of Karlsruhe. From 2003 to 2008, he was a Professor with the Department of Electrical Engineering and Information Technology, Technische Universität München, Germany. His research interests include image processing, automated visual inspection, information fusion, measurement technology, pattern recognition, and communications. 